\title{
Lipoid proteinosis: a first report of mutation Val10Gly in the signal peptide of the ECM1 gene
}

\author{
Dominik Ludew ${ }^{1}$, Katarzyna Wertheim-Tysarowska², Katarzyna Budnik¹, Alicja Grabarczyk², Cezary Kowalewski ${ }^{3}$, \\ Monika Kapińska-Mrowiecka ${ }^{1}$
}

${ }^{1}$ Department of Dermatology, Stefan Zeromski Krakow Municipal Hospital, Krakow, Poland ${ }^{2}$ Clinical Genetics Department, Institute of Mother and Child, Warsaw, Poland

${ }^{3}$ Department of Dermatology and Immunodermatology, Medical University of Warsaw, Warsaw, Poland

Adv Dermatol Allergol 2018; XXXV (2): 208-211 DOI: https://doi.org/10.5114/ada.2018.75245

Lipoid proteinosis is a rare autosomal recessive genodermatosis which affects approximately one in 300000 people, characterized by scarring and deposition of an amorphous material in the skin, mucosae membranes and viscera. Hyaline-like material is deposited around capillaries and adnexa causing basement membrane thickening [1]. Over 300 cases have been reported worldwide, including South African descendants of German or Dutch immigrants (due to the founder effect) and in the eastern Mediterranean region of Turkey (due to cultural allowance for consanguineous marriages) [2]. The disease typically follows a slowly progressive, yet often benign, course and appears to invariably manifest in the first 2 years of life with hoarseness and skin lesions. Virtually any organ may be involved, but involvement of internal organs rarely leads to deleterious consequences. Lifespan is otherwise normal but disease could cause psychological distress and diminished quality of life [3].

Mutations in the gene encoding extracellular matrix protein 1 (ECM1) on band 1q21 were identified as the cause of lipoid proteinosis in 2002 [4] and were located mostly in exons 6 and 7 (exon 7 mutations - milder, 6 - more severe form of the disease) [5]. Only three mutations have been described either in the initial first two exons or in the first intron of the ECM1 gene [6].

Patient concerns: a 9-year-old girl was admitted to the ENT department of the hospital. The main complaint from the parents was multiple nodular lesions affecting mucosa of gums and lips causing feeding difficulties and cosmetic defects.

Clinical findings: Physical examination on admission revealed skin-colored/yellowish waxy multiple papules along eyelid borders causing an irregular eyelash line; waxy papules grouping into plaques in the mucous membrane of the gums and lips. Dermal involvement was present predominantly affecting the skin of the forehead, characterized by atrophic scaring of the middle face and focal cicatricial alopecia (Figure 1 A). A low, hoarse vocal tone was evident. According to the mother, papular facial and pectoral skin lesions appeared in the $2^{\text {nd }}$ year of life with the subsequent evolution of papules into atrophic scars. Atopic dermatitis was initially diagnosed. Symptoms have gradually worsened over several years. She was frequently hospitalized in pediatric wards, humoral immunodeficiency was excluded when the girl was 5 year old but the lgE level $(201 \mathrm{U} / \mathrm{ml})$ was elevated. Skin allergy tests for pteronyssinus farinae, candida albicans, and alternaria antigens were positive. Lymphocytic subpopulations revealed a decreased CD4/CD8 ratio (CD3+ $59 \%(1711 / \mu \mathrm{l}), \mathrm{CD} 4+29 \% \downarrow(812 / \mu \mathrm{l}), \mathrm{CD} 8+26 \%(728 /$ $\mu l), C D 19+16 \% \downarrow(448 / \mu l), N K 24 \% \uparrow(672 / \mu l))$. Mitogen activated lymphocyte proliferation assays were normal. Anti-neutrophil cytoplasmic, against parietal cells, islet cells, endomysial, smooth muscle cells IgA and IgG, transglutaminase IgA, IgG antibodies were negative. Serum protein electrophoresis revealed an increased percentage of globulin $\alpha 13.7 \%, \alpha 213.2 \%$ and $\gamma$-globulins $18.7 \%$ and decreased albumin $54.1 \%$ (total protein concentration $77.8 \mathrm{~g} / \mathrm{l})$. Abdominal ultrasound disclosed the right renal pelvis (enlarged to $10 \mathrm{~mm}$ ), partially extrarenal with calyces dilated to $6 \mathrm{~mm}$.

Diagnostic focus and assessment: based on clinical features, lipoid proteinosis was diagnosed. Confirmatory mucosal biopsy with hematoxylin-eosin (Figure 1 C; upper slides) and periodic acid-Schiff (PAS), silver (Figure $1 \mathrm{C}$; left lower slide and Congo Red staining (Figure $1 \mathrm{C}$; right lower slides) revealed deposits of amorphous eosinophilic hyaline material localized predominantly in the subepidermal layer, also around capillaries (CD 34+) and skin appendages. The substance was PAS positive,

Address for correspondence: Dominik Ludew MD, PhD, Department of Dermatology, Stefan Zeromski Krakow Municipal Hospital, Os. Na Skarbie 66, 30-376 Krakow, Poland, phone: +48 693340 892, e-mail: dludew@interia.pl

Received: 5.11.2017, accepted: 12.11.2017. 
slightly silver staining, Congo Red positive but without apple green birefringence in the polarized light.

Additional tests aimed at detection of the visceral involvement were proposed: serum protein electrophoresis, abdominal ultrasound, neurological consultation with computed tomography (CT) scan and electroencephalography (EEG), and ophthalmologist consultation. All tests proved to be normal.

At the second consultation, the patient's 11-year-old sister was present. Lipoid proteinosis symptoms were evident but there was a considerable variation in clinical features between the affected patients. In the older sister, waxy papules in the mucous membrane of gums and lips were smaller, more noticeable, not grouping, causing minor complaints (Figure 1 B). Dermal involvement predominantly affecting the skin of the forehead was less severe, focal cicatricial alopecia was more diffuse. A low, hoarse vocal tone and multiple papules along the eyelid borders were also evident. She was frequently hospitalized in pediatric wards because of recurrent laryngitis. The celiac disease, atopic dermatitis, asthma were consequently suspected but humoral immunodeficiency was excluded. Laboratory tests revealed an elevated serum potassium level $(5.5 \mathrm{mmol} / \mathrm{l})$, serum protein electrophoresis showed an elevated total protein concentration $(81 \mathrm{~g} / \mathrm{l})$. Erythrocyte sedimentation rate (ESR) proved also to be elevated 40/80 (after 1 and 2 h). An EEG was conducted in wakefulness and sleep when the girl was 4 years old because of a hyperkinetic disorder and emotional disturbances but an EEG record revealed no pathological findings. On CT scans, pathognomonic parahippocampal bean-shaped calcifications present in up to $70 \%$ of the affected people were excluded. Parahippocampal calcifications could cause seizures and behavioral changes (difficulties in anger control and hyperkinetic disorder) in the affected individuals [7].

On consultation, the family history was investigated. Parents proved to be consanguineous - second cousins (Figure $1 \mathrm{D})$.

Examining genomic DNA by direct Sanger sequencing from affected and unaffected individuals in the studied family revealed homozygous substitution Val10Gly; (NP_004416.2:p.Val10Gly; NM_004425.3:c.29T>G) in affected children, whose both parents were confirmed to be heterozygous carriers (Figure $1 \mathrm{E}$ ). The Clustal X analysis was performed to check evolutionary conservation of novel mutations, the SSF (Splicing Sequences Finder), NNSPLICE (Splice Site Prediction by Neural Network) and HSF (Human Splicing Finder) software was used to evaluate in silico the potential effect of novel variants on splicing aberration and PolyPhen-2 to evaluate protein structure distortion. In silico analysis results showed results consistent with the pathogenic role of the reported mutation (supplementary materials). This is the first report of a missense mutation within the signal peptide domain of ECM1 causing lipoid proteinosis.
ECM1protein has at least four splice variants: ECM1a (540 amino acids; Figure 1 F), ECM1b (415 amino acids), ECM1c (567 amino acids according to current data from UniProt database or 559 amino acids as described previously in the literature [8]), and a short splicing variant (170 amino acids) [6]. ECM1a is the most widely expressed splice variant in various tissues including the skin, liver, small intestines, lung, ovary, prostate, testis, skeletal muscle, pancreas, kidney, placenta, and heart; while ECM1b has a much more restricted expression pattern, being detectable only in tonsils and keratinocytes. The full pattern of ECM1c expression has yet to be determined; however, in the skin it accounts for approximately $15 \%$ of total ECM1 RNA. ECM1 contains a signal peptide of 19 amino acids followed by four functional domains: a cysteine-free $\mathrm{N}$-terminus, two tandem repeats, and a C-terminus. The latter three domains contain 29 cysteine residues capable of forming protein double loops facilitating protein-protein interactions.

Since the first report of mutations in the ECM1 gene in six individuals with LP in 2002, 56 different pathogenic mutations have been published [8]. Over half of all mutations are located within exons 6 or 7 [6]. These include only 9 missense variants, 18 nonsenses, 7 splicing variants and 20 in- or out-of-frame insertions or deletions $<100$ bp and 2 deletions exceeding 1000 bp. Only three mutations have been described either in the initial first two exons or in the first intron of the ECM1 gene. Two of them are splicing mutations located in an invariant GT donor splice site of intron 1, while p.Val10Gly is not only the single variant identified in the first exon, but also the only one locating in a signal peptide.

Location and secretion of majority of eukaryotic proteins depends on signal peptides, which direct protein to cellular organelles or to translocon. Signal peptides are rich in hydrophobic amino acids that form a single $\alpha$-helix. In addition, many signal peptides begin with a short positively charged stretch of amino acids facilitating docking to ER. Mutations in a positively charged or hydrophobic region could cause inability to bind SRP-signal peptideribosome complex to ER resulting in intracellular protein retention. A similar mechanism has been recently reported for the COL5A1 signal peptide missense mutation Lys25Arg in the hydrophobic core as a cause of classic Ehlers-Danlos syndrome [9]. Missense mutations involving hydrophobic core of the signal peptide have been shown to stop protein secretion, cause defective protein folding and aggregation. Moreover, despite the fact that initial protein synthesis could take place, rapid product degradation in proteasome could result in non-detectable protein extra- and intracellular level, observed in individuals afflicted by mutations in the signal peptide [10].

Although examples of a number of other inherited disorders caused by alterations in targeting signals have already been reported, some authors indicate that the total number of mutations affecting signal peptides is under- 

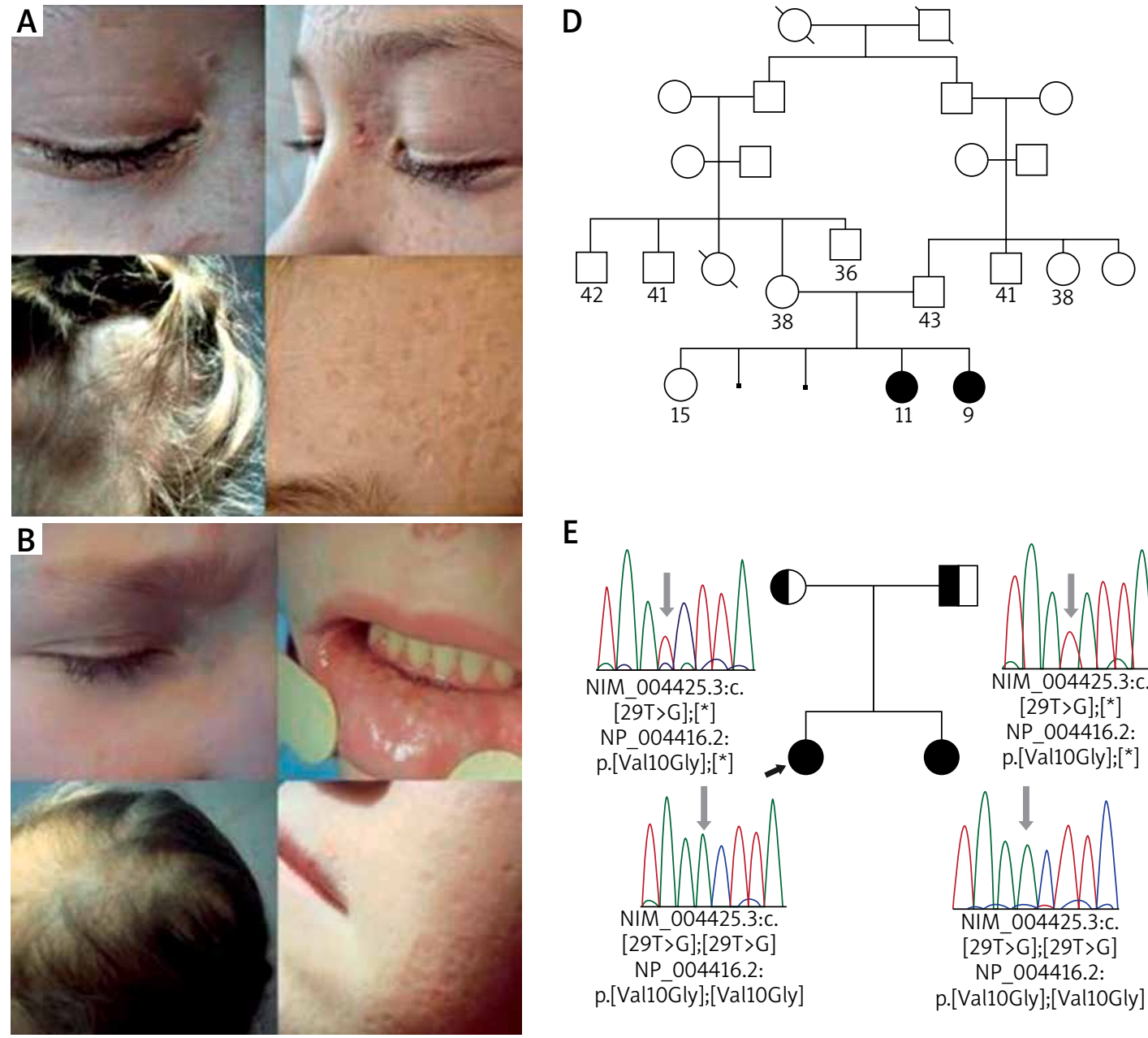

E
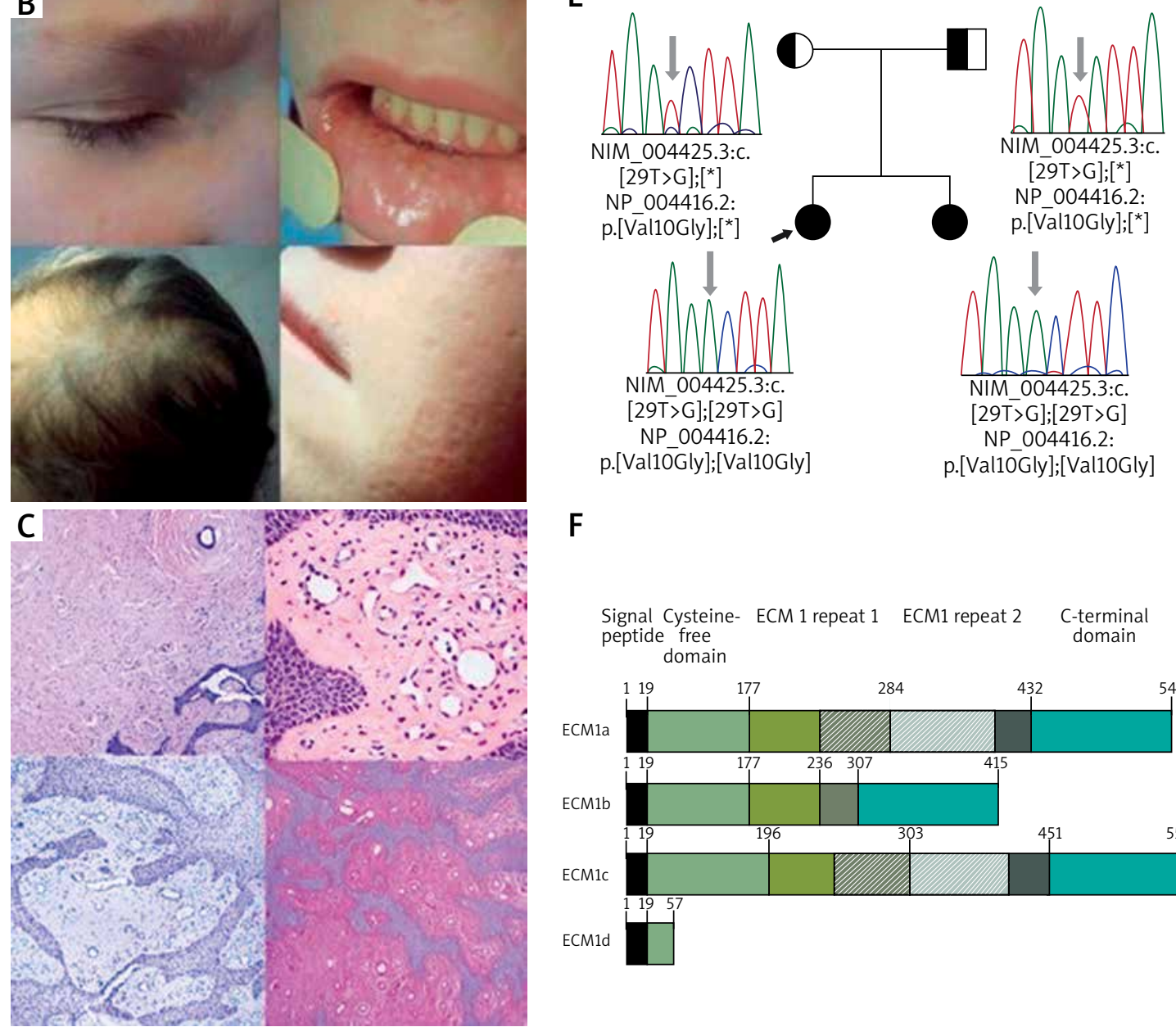

p.[Val10Gly];[Val10Gly]

p.[Val10Gly];[Val10Gly]

\section{$\mathrm{F}$}

Signal Cysteine- ECM 1 repeat 1 ECM1 repeat 2 C-terminal peptide free domain

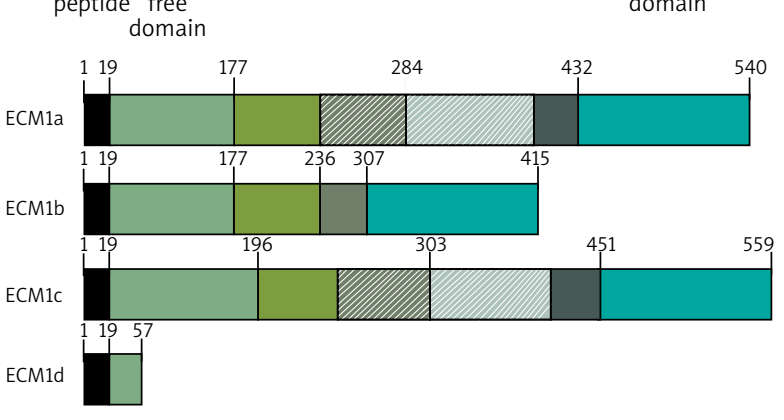

Figure 1. A - Clinical findings in 9-year-old patient on the left panel, B - clinical findings in 11-old-sister of the patient, $\mathbf{C}$ - confirmatory mucosal biopsy with haemathoxylin-eosin staining (upper slides) and specific silver staining (lower left slide) and Congo Red staining (lower right slide), D - genogram of the investigated family, E - electropherograms of 29T>G mutations of the investigated family. Arrows denotes mutated bases, F - schematic representation of ECM 1 and its four splice variants. ECM1 protein is divided in a signal sequence (19 aa) (black box) and four different domains based on the presence or absence of cysteines: an N-terminal cysteine-free domain (white box), two tandem repeats (green and gray box), and a C-terminal region (blue box). ECM1c differs from ECM1a containing 19 aa encoded by exon 5a, ECM1b results from an alternative trancript caused by splicing out exon 7 (shaded black). ECM1d encodes a truncated protein composed of 57 aa containing exon 1 , exon 2 and a part of exon 3 (13) 
estimated. Missense mutations are valuable predictors of functional consequences of the modified protein function and activity.

The missense mutation in the first exon of ECM1 p.Val10Gly was previously discovered in a Polish family in combination with additional translation-affecting splicing mutation IVS1+1G $>$ A. This report was not published hence the expected severe clinical course of the disease is not proven [6]. However, another mutation of the same locus- IVS1+1G>C was published in a large family of Arabian origin in Israel with relatively severe skin involvement [11].

Noteworthy a severe course of the disease is reported in cases where mutations affecting $\mathrm{N}$-terminal as well as C-terminal fragments of the gene were present. Of note, affected Indian individuals have been found to have homozygous nonsense mutations (p.Arg476Ter, p.Arh481Ter) close to the 3' end of the gene in exon 10 (Chan and McGrath, unpublished data) but the LP phenotypes show no differences from other affected cases and were certainly not milder [12].

Conversely, the clinical outcome of LP in the siblings harboring the same homozygous mutations presented herein varies. Thus, the case presented by us not only provides further data on the ECM1 mutation spectrum, but also supports the role of the signal peptide sequence for proper ECM1 functioning and clearly indicates that the genotype is not the single factor influencing the phenotype [13].

\section{Acknowledgments}

The authors have obtained written consent from the parents of the presented children to report personal data including images and genogram of the family.

\section{Conflict of interest}

The authors declare no conflict of interest.

\section{References}

1. Sercu S, Zhang M, Oyama N, et al. Interaction of extracellular matrix protein 1 with extracellular matrix components: ECM1 is a basement membrane protein of the skin. J Invest Dermatol 2008; 128: 1397-408.

2. Lee MY, Wang HJ, Han Y, et al. Lipoid proteinosis resulting from a large homozygous deletion affecting part of the ECM1 gene and adjacent long non-coding RNA. Acta Derm Venereol 2015; 95: 608-10.

3. Kaya TI, Gunduz O, Kokturk A, et al. A life-threatening exacerbation of lipoid proteinosis. J Eur Acad Dermatol Venereol 2002; 16: 286-8.

4. Hamada T, McLean WH, Ramsay M, et al. Lipoid proteinosis maps to 1q21 and is caused by mutations in the extracellular matrix protein 1 gene (ECM1). Hum Mol Genet 2002; 11: 833-40.

5. Lupo I, Cefalu AB, Bongiorno MR, et al. A novel mutation of the extracellular matrix protein 1 gene (ECM1) in a patient with lipoid proteinosis (Urbach-Wiethe disease) from Sicily. Br J Dermatol 2005; 153: 1019-22.

6. Chan I, Liu L, Hamada T, et al. The molecular basis of lipoid proteinosis: mutations in extracellular matrix protein 1. Exp Dermatol 2007; 16: 881-90.

7. Thornton HB, Nel D, Thornton D, et al. The neuropsychiatry and neuropsychology of lipoid proteinosis. J Neuropsychiatry Clin Neurosci 2008; 20: 86-92.

8. Youssefian L, Vahidnezhad H, Daneshpazhooh M, et al. Lipoid proteinosis: phenotypic heterogeneity in Iranian families with c.507delT mutation in ECM1. Exp Dermatol 2015; 24: 220-2.

9. Symoens S, Malfait F, Renard M, et al. COL5A1 signal peptide mutations interfere with protein secretion and cause classic Ehlers-Danlos syndrome. Hum Mutat 2009; 30: E395-403.

10. Vijayasarathy C, Sui R, Zeng Y, et al. Molecular mechanisms leading to null-protein product from retinoschisin (RS1) signal-sequence mutants in $\mathrm{X}$-linked retinoschisis (XLRS) disease. Hum Mutat 2010; 31: 1251-60.

11. Horev L, Potikha T, Ayalon S, et al. A novel splice-site mutation in ECM-1 gene in a consanguineous family with lipoid proteinosis. Exp Dermatol 2005; 14: 891-7.

12. Nasir M, Latif A, Ajmal M, et al. Molecular analysis of lipoid proteinosis: identification of a novel nonsense mutation in the ECM1 gene in a Pakistani family. Diagn Pathol 2011; 6: 69.

13. Atlas of Genetics and Cytogenetics in Oncology and Haematology; Schematic representation of ECM1 and its four splice variants. Accessed 20 July 2017 [Available from: http:// atlasgeneticsoncology.org/Genes/ECM1ID40398ch1q21.html. 